\title{
Electroless coating of carbon nanotube/graphene nanoplatelets for reinforcing $\mathrm{FeCo}$ alloy
}

\author{
Amar J. Albaaji ${ }^{1}$, Ahmed Mudhafar Hashim ${ }^{1}$, Heider Yasser Thamir ${ }^{1}$ \\ ${ }^{1}$ Material department, College of Engineering, University of Al-Qadisiyah, Iraq
}

\begin{abstract}
Electroless coating process was used to coat graphene nanoplatelets (GNPs) and carbon nanotubes (CNTs), which were dispersed for 1 vol.\% by planetary ball milling in FeCo alloy. Spark plasma sintering (SPS) parameters of heating rate of $50{ }^{\circ} \mathrm{C} / \mathrm{min}$, sintering pressure of $50 \mathrm{MPa}$ and sintering temperature of $1100{ }^{\circ} \mathrm{C}$ without dwelling were used for sintering. Tensile test was used to evaluate the mechanical properties for the sintered materials. The variations in microstructure and phases were analysed by Optical microscope, Scanning electron microscope, Transmission electron microscope, X-ray diffraction and Raman spectroscopy tests. Coating process was effective in improving the tensile strength and yield strength in CNTs composite; however, the ductility was decreased after coating for the reinforcements.
\end{abstract}

Keywords: $\quad$ Electroless coating, Graphene nanoplatelets, Mechanical properties

\section{Corresponding Author:}

Amar J. Albaaji

Material department, College of Engineering, University of Al-Qadisiyah, Iraq.

E-mail: amar.jabar@qu.edu.iq

\section{Introduction}

FeCo alloy is one of the most important soft magnetic materials, due to its high magnetic properties. These properties are required in more electrical aircraft applications. However, the mechanical properties of the alloy are not suitable for such applications [1,2]. Different methods were used to develop the mechanical properties of the FeCo alloy, which include alloying with third element, heat treatment, and plastic deformation. Despite, the mechanical properties are improved by these processes, the magnetic properties are deteriorated in these processes, in addition to instability for mechanical properties at elevated temperature [3]. Innovation of carbonaceous nanomaterials such as graphene and carbon nanotube open new widow to develop material by nanocomposites. However, obtaining uniform dispersion for carbonaceous nanomaterials is extremely challenge [4]. Moreover, maintain the nanostructure through the dispersion and sintering processes is very difficult. Spark plasma sintering is new sintering process with many advantages over other sintering processes, such as fast heating rate, short time for sintering, binderless process, application of pressure during sintering, cleaning effect for grain boundaries, maintain nanostructure [5]. Recently, the mechanical properties of $\mathrm{FeCo}$ alloy have been improved by spark plasma sintering alone and by addition graphene or carbon nanotube with aid spark plasma sintering [6,7]. Coating for reinforcement was also used to improve the properties of FeCo alloy $[8,9]$. However, to the best of our knowledge there is no such study for comparison the influence of coating CNT/ GNP on the mechanical properties of FeCo alloy. The main aim of the current study is to find if there is any possibility to improve the properties of FeCo alloy through improving the dispersion and the interface bonding by coating the reinforcing carbonaceous nanomaterial. 


\section{Experimental work}

\subsection{Sensitisation and activation}

The CNTs and GNPs were sensitized by dispersion in the sensitisation solution, which was prepared by mixing $3.39 \mathrm{~g}$ of $\mathrm{SnCl}_{2} . \mathrm{H}_{2} \mathrm{O}$ in $1.5 \mathrm{ml}$ of $\mathrm{HCl}$, the volume was made up $150 \mathrm{ml}$ by de-ionised water and stirred magnetically for $30 \mathrm{~min}$. After that, $0.06 \mathrm{~g}$ of the functionalised CNTs or GNPs were introduced into sensitisation solution and mixed by magnetic stirrer at room temperature for $30 \mathrm{~min}$ to enrich the surface of carbonaceous nanomaterials with tin atoms. Then the sensitized particles were washed thoroughly in deionised water with aid centrifuge machine to separate the particles from the de-ionised water, followed by drying on hot plate at $100{ }^{\circ} \mathrm{C}$ for $2 \mathrm{~h}$. The activation solution was prepared by mixing $0.075 \mathrm{~g}$ of $\mathrm{PdCl}_{2}$ and 3.9 $\mathrm{ml}$ of $\mathrm{HCl}$ in $150 \mathrm{ml}$ of de-ionised water by magnetic stirrer for $30 \mathrm{~min}$ [10], then the sensitised particles were introduced to activation solution the particles were magnetically stirred for 30 minutes at room temperature, then the particles were washed thoroughly and dried as above-mentioned procedure.

\subsection{Electroless coating process}

The chemical composition of $300 \mathrm{ml} \mathrm{Ni-P}$ coating bath is presented in Table 1 [11]. The chemicals were mixed in $300 \mathrm{ml}$ of de-ionised water; the solution was magnetically stirred to completely dissolve the salts and to achieve the desired $\mathrm{pH}$ value. Then the sensitised and activated particles were introduced to plating bath and stirred by ultrasonic probe for $10 \mathrm{~min}$. Following that the coated particles were extracted from bath by centrifuge, thoroughly washed and dried.

Table 1. Chemical composition of coating bath

\begin{tabular}{ll}
\hline Chemical & Amount \\
\hline Nickel sulphate (NiSO4.7H2O) & $7.5 \mathrm{~g}$ \\
Sodium phosphate $(\mathrm{NaH} 2 \mathrm{PO} 2 . \mathrm{H} 2 \mathrm{O})$ & $4.5 \mathrm{~g}$ \\
Tri-sodium citrate $(\mathrm{Na} 3 \mathrm{C} 6 \mathrm{H} 5 \mathrm{O} 7 . \mathrm{H} 2 \mathrm{O})$ & $1.5 \mathrm{~g}$ \\
Ammonium chloride $\mathrm{NH} 4 \mathrm{Cl}$ & $18 \mathrm{~g}$ \\
Ammonium hydroxide $(2.0 \mathrm{M}$ ammonia water $)$ & to adjust $\mathrm{pH}$ to 8.25 \\
Temperature & $25^{\circ} \mathrm{C}$ \\
\hline
\end{tabular}

\subsection{Preparing composites}

The flaked FeCo alloy powder was prepared by planetary ball milling machine under argon atmosphere. Fifty grams of FeCo alloy was ball milled at BPR of $\sim 2: 1$ for $6 \mathrm{~h}$, with $8 \mathrm{ml}$ of isopropanol alcohol as process control agent (PCA), for operating conditions of a milling speed of $300 \mathrm{rpm}$, to avoid the overheating during process a $15 \mathrm{~min}$ work and $10 \mathrm{~min}$ pause were selected. To prepare the composites, twenty grams of flaky FeCo powder was mixed with the 1 vol.\% of coated carbonaceous nanomaterials by ball milling at ball to powder ratio of $1: 1$ for $1 \mathrm{~h}$ under argon atmosphere and using $6 \mathrm{ml}$ of isopropanol alcohol as a PCA. The used operation parameters for ball milling were $400 \mathrm{rpm}$ for $30 \mathrm{~min}$ work and $15 \mathrm{~min}$ pause.

\section{Results and discussion}

\subsection{TEM of as received CNT and GNP}

The morphology of CNT and GNP are presented in Fig.1, which shows wide variation in dimensions and some defect from fabrication process as shown by arrows. 


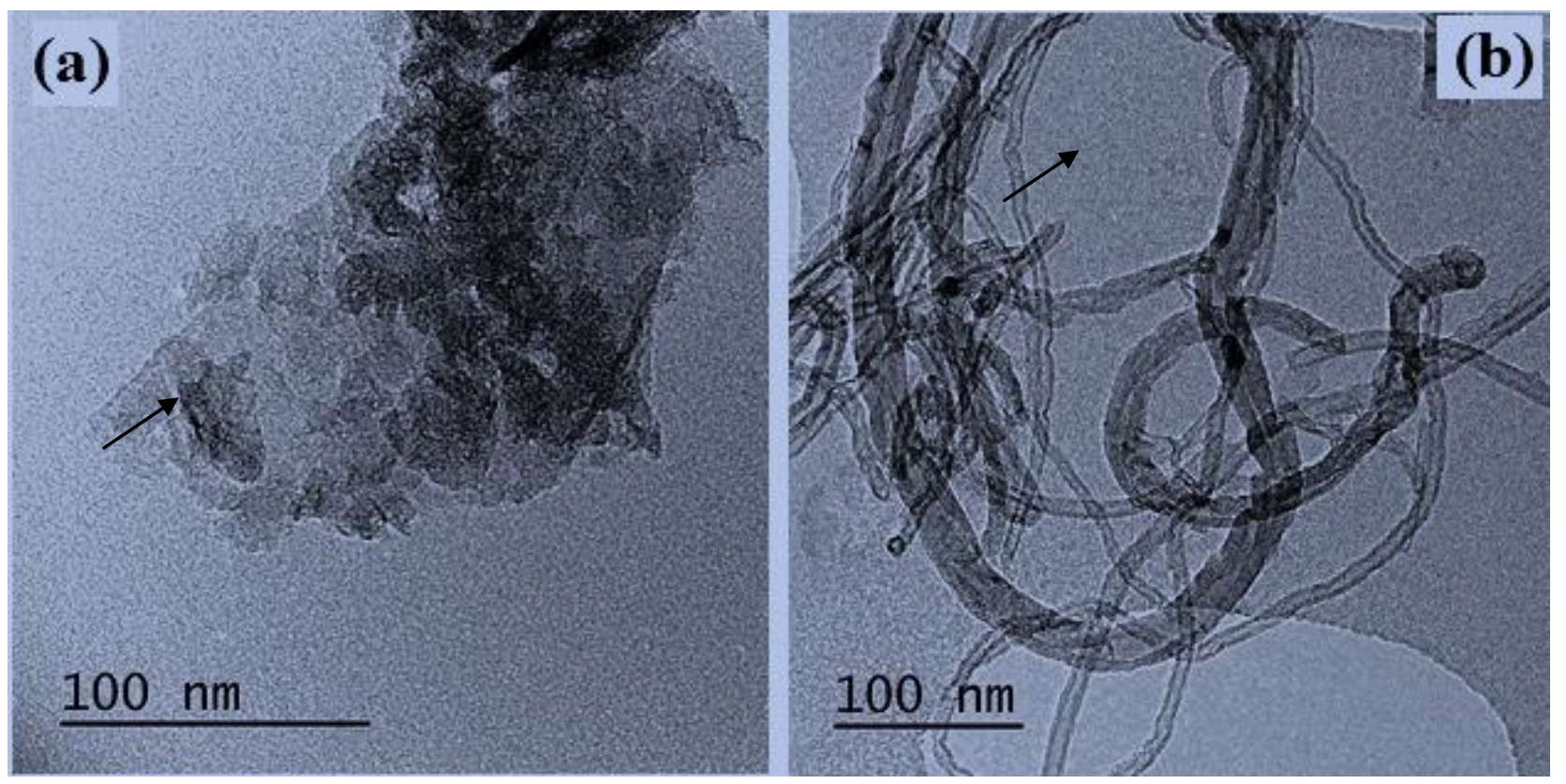

Fig. 1. Transmission Electron Microscope (TEM) of; (a) GNP and (b) CNT

After coating process, EDS analysis was performed Fig.2, which shows a composition from $\mathrm{Ni}$ and $\mathrm{P}$ in coating layer. CNTs are embedded within coating materials, therefore, the subsequent dispersion process was performed by ball milling to breakdown the agglomeration after coating.

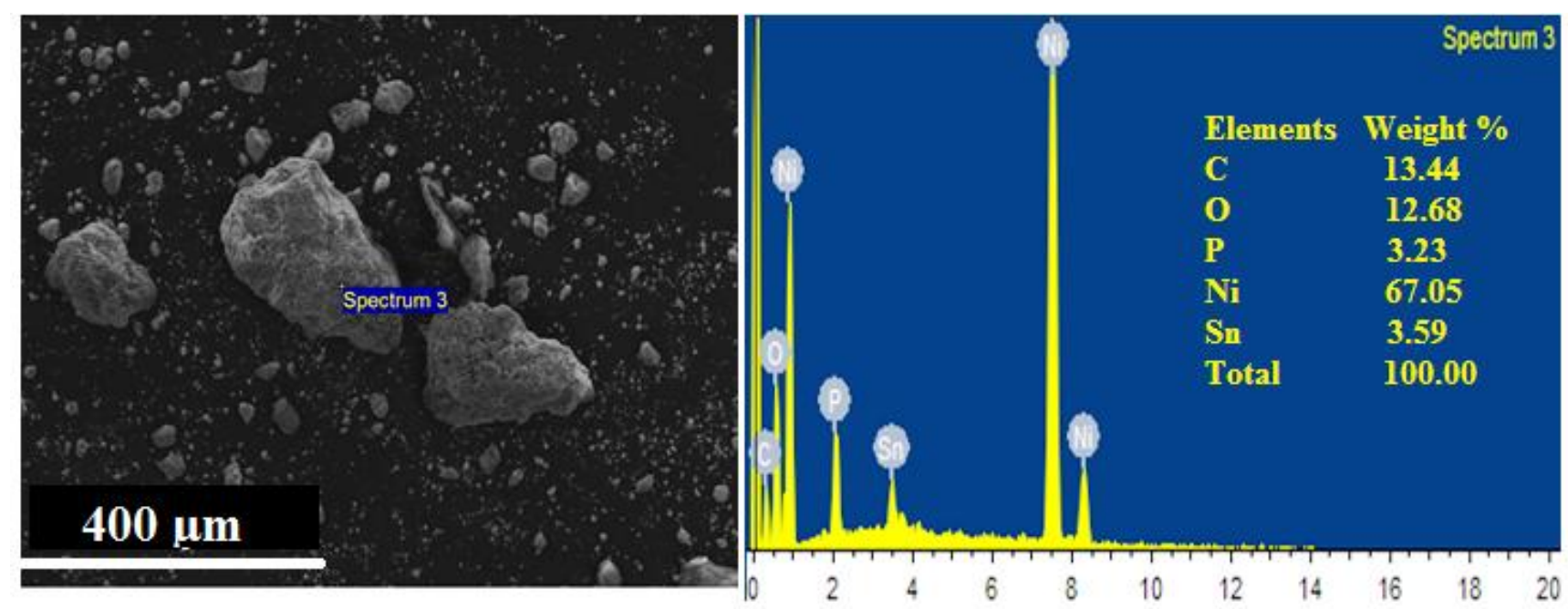

Fig. 2. Energy-dispersive X-ray spectroscopy (EDS) analysis of Ni-P coating CNT

\subsection{Relative density}

The relative densities of the sintered materials are shown in Table 2. The highest values for relative density are observed in CNT composites. This may be due less agglomeration in CNT composites as compared to GNP composites. The relative density was decreased in coated reinforcement composites, this can be rationalised to the observed agglomeration in (Fig.2) after coating processes. 
Table 2. Relative densities of the sintered materials

\begin{tabular}{lc}
\hline Sample details & Relative density (\%) \\
\hline As received FeCo alloy & 98.99 \\
Ball milled FeCo alloy & 98.62 \\
1 vol.\% bare CNT-FeCo composite & 100.39 \\
1 vol.\% Ni-P coated CNT-FeCo composite & 99.09 \\
1 vol.\% bare GNP-FeCo composite & 98.37 \\
1 vol.\% Ni-P coated GNP-FeCo composite & 97.06 \\
\hline
\end{tabular}

\subsection{Mechanical properties}

The stress-strain curves of the as received, ball milled FeCo alloy and the composites of coated and uncoated carbonaceous reinforcements are shown in Fig.3. The ultimate tensile strength, yield strength, the elongation and hardness measurements of the sintered materials are summarised in Table 3. An improvement in both ultimate strength and yield strength was observed for both uncoated and coated CNT and GNP composites in comparison to as received FeCo alloy. The highest value was obtained in 1 vol. \% Ni-P coated CNT-FeCo composite, when the ultimate strength was increased from $400 \pm 20 \mathrm{MPa}$ to $728 \pm 66 \mathrm{MPa}$ and yield strength from $340 \pm 0.5 \mathrm{MPa}$ to $645 \pm 30.2 \mathrm{MPa}$ in comparison to as received FeCo alloy. The highest density was achieved in CNT-composite, which has an influence on mechanical properties. This may be due to improve the interface bonding after coating the reinforcement and to the role of $\mathrm{Ni}$ in formation stable precipitates, refine grain size, and decrease the degree of long range ordering [3, 9, 12]. All those factors work together in obtaining the highest value in the properties after coating CNT by Ni-P. It has been reported that the interfacial bonding is improved after coating the reinforcement by Ni-P layer in FeCo alloy composites. Due to the chemical reaction for the coating layer with matrix and limited reaction with the reinforcement, two regions could be formed in coating layer, one with high Ni content close to reinforcement and other with high Fe content close to the matrix [8]. These reactions improve the interfacial bonding by forming intermetallic compound between $\mathrm{Fe}$ and $\mathrm{Ni}$ and also cause deviation in chemical composition of FeCo alloy, the subsequent of that is the improved mechanical properties. The properties were decreased in Ni-P coated GNP composite. It is suggested that may be the non-uniformity in coating for reinforcement may be caused the different in properties of the composites. Further, the relative density was more decreased in GNP-composites due to more influence of two-dimension GNP on agglomeration, which caused drop in properties. It is found that Ni-P coated reinforcement was only effective in improving the mechanical properties when the coating layer is thick enough. However, the increase in coating layer thickness causes an increase in coercivity value due to more precipitations [13]. Despite, the enhancement in elongation of bare carbonaceous reinforcement, coating of GNP and CNT by Ni-P leads to decrease in elongation value especially in CNT-composite. This can be attributed to agglomeration after coating processes. The hardness values were increased for all samples in comparison to as received monolithic FeCo alloy, the significant increase in hardness was from $255.52 \pm 8.51$ in as received monolithic FeCo alloy to $323.5 \pm 1.48$ for 1 vol. \% bare GNP-FeCo composite, which reflect the effective role for GNP in improving the hardness in comparison to CNT due to the two-dimensional morphology. However, harness value of coated GNP-FeCo composite was declined due to including porosity in microstructure as evidence from the lowest value of the relative density Table 2 . The increased hardness values in composites as compared to the as received can also rationalized to the role of CNT and GNP in refining the microstructure. 


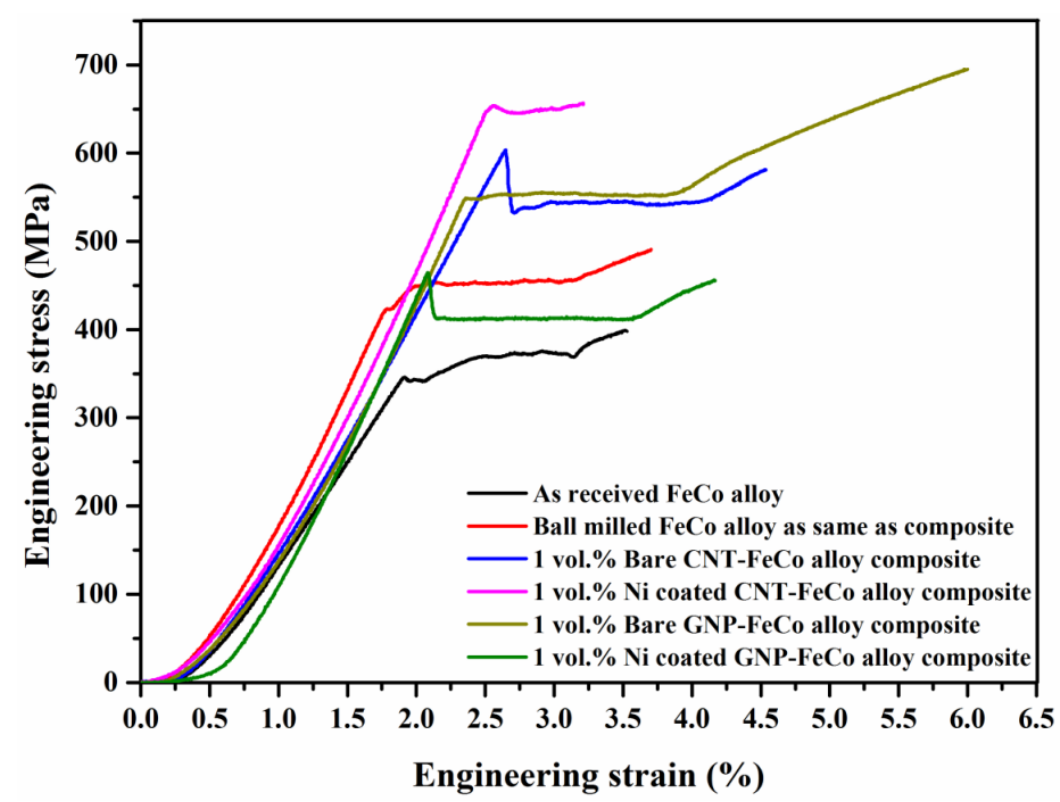

Fig. 3. Tensile stress-strain curves of monolithic FeCo alloy and composite materials

Table 3. Mechanical properties of FeCo alloy and composite of bare and coated CNT and GNP

\begin{tabular}{|c|c|c|c|c|}
\hline Sample details & $\begin{array}{c}\text { Ultimate } \\
\text { strength (MPa) } \\
\pm 5 \%\end{array}$ & $\begin{array}{l}\text { Yield strength } \\
(\mathrm{MPa}) \pm 5 \%\end{array}$ & $\begin{array}{l}\text { Elongation } \\
(\%) \pm 5 \%\end{array}$ & $\begin{array}{c}\text { Hardness } \\
(\text { HVN }) \pm 3 \\
\%\end{array}$ \\
\hline As received FeCo alloy & 400 & 340 & 3.5 & 255.5 \\
\hline Ball milled FeCo alloy & 516 & 450 & 3.5 & 304.8 \\
\hline 1 vol.\% bare CNT-FeCo composite & 553 & 525 & 4.1 & 307.8 \\
\hline $\begin{array}{l}1 \text { vol.\% Ni-P coated CNT-FeCo } \\
\text { composite }\end{array}$ & 728 & 645 & 2.7 & 321.7 \\
\hline 1 vol.\% bare GNP-FeCo composite & 651 & 555 & 5.0 & 323.5 \\
\hline $\begin{array}{l}1 \text { vol. } \% \text { Ni-P coated GNP-FeCo } \\
\text { composite }\end{array}$ & 430 & 415 & 3.9 & 281.7 \\
\hline
\end{tabular}

\subsection{Fracture surface}

Figure 4 shows the fracture surface of as received FeCo alloy, ball milled FeCo alloy and Ni-P coated GNP and CNT-FeCo alloy composites. Due to the weakness in intergranular bonding in FeCo alloy, the alloy normally fails by intergranular fracture mode [2]. The sintered as received and ball milled monolithic FeCo alloy shows transgranular fracture mode, which is changed to intergranular mode after embedding the coated carbonaceous nano reinforcement in FeCo alloy. This can be rationalised to the influence of agglomerated particles at grain boundaries on bonding or may be due to contamination by chemical materials after coating. 

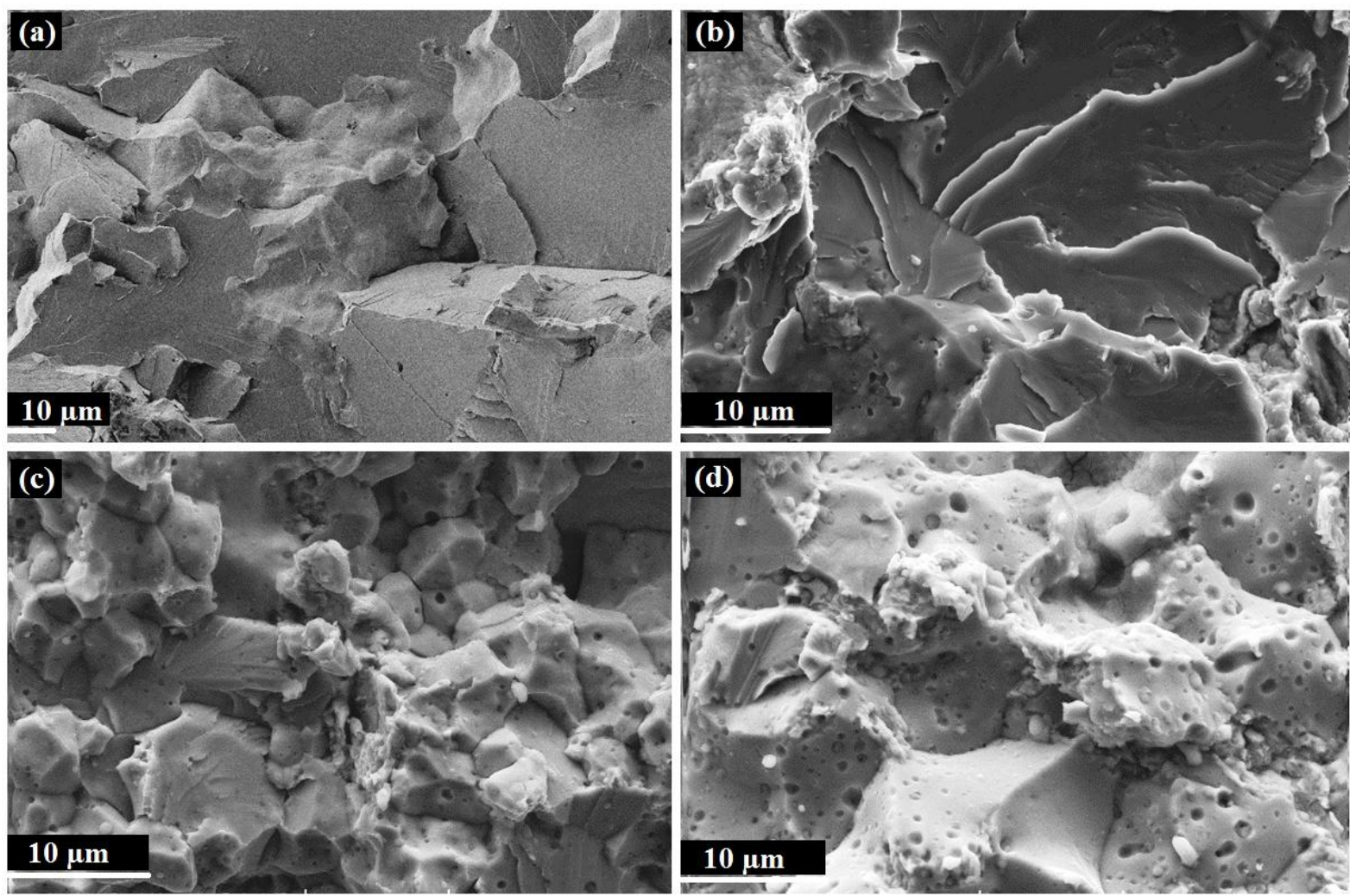

Fig. 4. Fractographic images of (a) As received FeCo alloy, (b) Ball-milled FeCo alloy for the same time as composite, (c) 1 vol.\% Ni-P coated CNT-FeCo alloy composite, (d) 1 vol.\% Ni-P coated GNP-FeCo alloy composite

\section{Conclusion}

1- Ni-P coating of CNT improves the strength in FeCo alloy composite, however it caused a decrease in elongation, this can be rationalised to inhomogeneous coating and agglomeration of particles during coating. 2- Bare GNP is very effective as compared to coated reinforcements in improving the elongation of FeCo alloy, this may be due to high surface area of GNP in bridging cracks.

3- The granular bonding is reduced due to the agglomeration for the coated carbonaceous nanomaterials at grain boundaries.

\section{References}

[1] Quigley RE: Proceedings of IEEE applied power electronics conference, San Diego, CA, March 1993, pp 906-911.

[2] Sundar RS, Deevi SC: Int Mater Rev, 2005, 3, vol 50, pp.157-192.

[3] Sourmail, T: Progress in Materials Science, 2005, 7, vol 50, pp.816-880.

[4] Chu, K. \& Jia, C.:Physica Status Solidi (A) Applications and Materials Science, 2014, 1,vol 211, pp.184190.

[5] Munir, Z., Anselmi-Tamburini, U., Ohyanagi, M: Journal of Materials Science. 2006, 3, vol 41, pp 763777.

[6] Albaaji, A. J., Castle, E. G., Reece, M. J., Hall, J. P., \& Evans, S. L.: Journal of Materials Science, 2017, 22, vol 52, pp.13284-13295.

[7] Albaaji, A. J., Castle, E. G., Reece, M. J., Hall, J. P., \& Evans, S. L.: Materials \& Design, 2017, vol 122, pp. 296-306. 
[8] Mani, M.K., Viola, G., Reece, M.J., Hall, J.P. and Evans, S.L.: Journal of Materials Science, 2014, 6, vol 49, pp. 2578-2587.

[9] Mani, M.K., Viola, G., Reece, M.J., Hall, J.P. and Evans, S.L.: Materials Science and Engineering: B, 2014, vol 188, pp. 94-101.

[10] Ang, L.M., Hor, T.A., Xu, G.Q., Tung, C.H., Zhao, S.P. and Wang, J.L.: Carbon, 2000, 3, vol 38, pp.363372.

[11] Kong, F.Z., Zhang, X.B., Xiong, W.Q., Liu, F., Huang, W.Z., Sun, Y.L., Tu, J.P. and Chen, X.W.: Surface and Coatings Technology, 2002, 1, vol 155, pp.33-36.

[12] Sundar, R.S. and Deevi, S.C.: Intermetallics, 2004, 7, vol 12, pp.921-927.

[13] Mani, M.K., Viola, G., Reece, M.J., Hall, J.P. and Evans, S.L.: Journal of Materials Science \&Engineering A, 2014, vol 592, pp.19-27. 